\title{
EQUICONTINUITY AND $n$-LENGTH
}

\section{EDWARD SILVERMAN ${ }^{1}$}

Let $(M, \rho)$ be a pseudo-metric space. We shall obtain a necessary and sufficient condition that a collection of curves can be parametrized in such a manner that the collection of parametrizations be equicontinuous. This result can be extended to the case where $\rho$ is a quasi-pseudo-metric. The $\mu$-length defined here differs inessentially from that originally defined by $M$. Morse in [M]. We also use ideas of $[\mathbf{F}]$ and $[\mathrm{S}]$.

Let $I=[a, b]$. If $u \in I$, then let $I_{u}=[a, u]$. If $(N, \sigma)$ is a pseudometric space and if $f: I \rightarrow N$, then let $\omega(f ; J)=\sup \{\sigma(f(u), f(v)) \mid u, v \in J\}$ whenever $J$ is an interval contained in $I$. Let $C(I)$ be the space of continuous functions on $I$ into $(M, \rho)$ metrized by $\sigma$ where $\sigma(x, y)$ $=\sup \{\rho(x(u), y(u)) \mid u \in I\}$.

For each positive integer $n$ and $x \in C(I)$, let

$$
\mu_{n} x=\sup \left\{\sum_{i=1}^{n} \rho\left(x\left(t_{i-1}\right), x\left(t_{i}\right)\right) \mid a \leqq t_{0} \leqq t_{1} \leqq \cdots \leqq t_{n} \leqq b\right\} .
$$

Since $x$ is continuous and $I$ is compact, the supremum is a maximum. Evidently $\mu_{n} \leqq \mu_{n+1}, \mu_{n} \leqq n \mu_{1}$ and $\left|\mu_{n} x-\mu_{n} y\right| \leqq 2 n \sigma(x, y)$.

Lemma 1. Let $A \subset C(I)$ be equicontinuous and $M_{n}=\sup \left\{\mu_{n} x \mid x \in A\right\}$. The $\lim _{n \rightarrow \infty} n^{-1} M_{n}=0$.

Proof. Let $\epsilon>0$. There exists $\delta>0$ such that $\omega(x ; J)<\epsilon$ whenever length $J=|J|<\delta$. Let $K>\delta^{-1}|I|$ and let $T_{j}, j=1,2, \cdots, K-1$, be points in $I$ which divide $I$ into $K$ equal subintervals. Let $t_{i} \in I$, $\mathrm{i}=0,1, \cdots, n$, and let $\left\{\sigma_{m}\right\}, m=0,1, \cdots, R=n+K-1$, be a nondecreasing arrangement of $\left\{t_{i}\right\} \cup\left\{T_{j}\right\}$. If $x \in A$ then

$$
\sum_{i=1}^{n} \rho\left(x\left(t_{i-1}\right), x\left(t_{i}\right)\right) \leqq \sum_{m=1}^{R} \rho\left(x\left(\sigma_{m-1}\right), x\left(\sigma_{m}\right)\right)<(n+K-1) \epsilon .
$$

If $x \in C(I)$ and $u \in I$ let $\phi_{n, x}(u)=\mu_{n} x \mid I_{u}$.

Lemma 2. If $n>2$, then $\phi_{n-2, x} \geqq(1-3 / n) \phi_{n, x}$.

Received by the editors September 5, 1967 and, in revised form, December 5, 1967.

1 This research was supported in part by National Science Foundation Grant GP-04088. 
Proof. It is sufficient to show that $\mu_{n-2} x \geqq(1-3 / n) \mu_{n} x$. There exists a nondecreasing sequence $\left\{t_{i}\right\}$ in $I$ such that $\sum_{i=1}^{n} a_{i}=\mu_{n} x$, where $a_{i}=\rho\left(x\left(t_{i-1}\right), x\left(t_{i}\right)\right)$. Let

$$
\begin{aligned}
& b_{i}^{j}=a_{i} \quad \text { if } i>j+1 \text { or } i<j-1 \text {, } \\
& =0 \quad \text { if } j-1 \leqq i \leqq j+1
\end{aligned}
$$

for $i=0,1, \cdots, n$ and $j=1,2, \cdots, n$.

If $A=\sum_{j=1}^{n} \sum_{i=1}^{n} b_{i}^{\prime}$, then

$$
\begin{aligned}
A & =\sum_{j=1}^{n-2} \sum_{i=j+2}^{n} a_{i}+\sum_{j=3}^{n} \sum_{i=1}^{j-2} a_{i} \\
& =\sum_{i=3}^{n} \sum_{j=1}^{i-2} a_{i}+\sum_{i=1}^{n-2} \sum_{j=i+2}^{n} a_{i} \\
& =\sum_{i=3}^{n}(i-2) a_{i}+\sum_{i=1}^{n-2}(n-i-1) a_{i} \\
& =(n-3) \sum_{i=1}^{n} a_{i}+a_{1}+a_{n} .
\end{aligned}
$$

Hence there exists $k$ such that $\sum_{t=1}^{n} b_{i}^{k} \geqq(1-3 / n) \mu_{n} x$. If $t_{k-1}$ and $t_{k}$ are deleted from $\left\{t_{i}\right\}$ and if the resulting nondecreasing sequence is labelled $\left\{\sigma_{j}\right\}, j=0,1, \cdots, n-2$, then

$$
\mu_{n-2} x \geqq \sum_{j=1}^{n-2} \rho\left(x\left(\sigma_{j-1}\right), x\left(\sigma_{j}\right)\right) \geqq \sum_{i=1}^{n} b_{i}^{k} \geqq(1-3 / n) \mu_{n} x .
$$

We now define a $\mu$-length on $C(I)$ by $\mu=\sum_{n=1}^{\infty} 2^{-n} \mu_{n}$. Thus $\mu_{1} \leqq \mu \leqq \mu_{1} \sum_{n=1}^{\infty} 2^{-n} n=2 \mu_{1}$. If $x \in C(I)$, let $\phi_{x}(u)=\mu x \mid I_{u}$. Evidently $2^{-k} \omega\left(\phi_{k, x} ; J\right) \leqq \omega\left(\phi_{x} ; J\right) \leqq 2 \omega(x ; J)$.

LEMMA 3. $\omega(x, J) \leqq \max \left\{6 n^{-1} \mu_{n} x, 2^{n+1} \omega\left(\phi_{x} ; J\right)\right\}$ for all $n$.

Proof. Let $u, v \in J$ with $u<v$ and let $2 \eta=\rho(x(u), x(v))$. If $\eta>3 n^{-1} \mu_{n} x$, then

$$
\phi_{n, x}(v)-\phi_{n, x}(u) \geqq \phi_{n-2, x}(u)+2 \eta-\phi_{n, x}(u) \geqq 2 \eta-3 n^{-1} \phi_{n, x}(u)>\eta .
$$

Hence $\eta<\omega\left(\phi_{n, x} ; J\right) \leqq 2^{n} \omega\left(\phi_{x} ; J\right)$.

CoRollary. A necessary and sufficient condition that $\phi_{x}$ be constant on $J$ is that $x$ be constant on $J$.

Let $C_{\mu}=\left\{X \in C([0,1]) \mid \phi_{X}(u)=(\mu X) u\right.$ for all $\left.u \in[0,1]\right\}$. Evidently $\omega\left(\phi_{X} ; J\right)=(\mu X)|J|$ and 


$$
2^{-1}(\mu X)|J| \leqq \omega(X ; J) \leqq \max \left\{6 n^{-1}\left(\mu_{n} X\right), 2^{n+2}\left(\mu_{1} X\right)|J|\right\}
$$

for all $n$ whenever $X \in C_{\mu}$.

Lemma 4. Let $A \subset C_{\mu}$ and $M_{n}=\sup \left\{\mu_{n} X \mid X \in A\right\}$. Then $A$ is equicontinuous if and only if $\lim _{n \rightarrow \infty} n^{-1} M_{n}=0$.

Proof. Choose $\epsilon>0$ and take $j$ so that $6 M_{j}<j \epsilon$. Let $\delta=\left[2^{j+2} M_{1}\right]^{-1} \epsilon$. If $u \leqq v<u+\delta$, then

$$
\rho(X(u), X(v)) \leqq \omega(X ;[u, v]) \leqq \max \left\{6 j^{-1} M_{j}, 2^{j+2} M_{1} \delta\right\}=\epsilon
$$

and the proof is complete because of Lemma 1.

Lemma 5. Let $X_{n} \in C_{\mu}$. Then $\lim _{n \rightarrow \infty} n^{-1}\left(\mu_{n} X_{n}\right)=0$ if and only if $\left\{X_{n}\right\}$ is equicontinuous.

Proof. If $\left\{X_{n}\right\}$ is equicontinuous, then

$$
0=\lim _{k \rightarrow \infty} k^{-1} \sup _{n} \mu_{k} X_{n} \geqq \lim _{k \rightarrow \infty} k^{-1}\left(\mu_{k} X_{k}\right) \geqq 0 .
$$

The proof in the other direction is like that of the preceding lemma.

If $x \in C(I)$ and $y \in C(J)$, let $D_{F}(x, y)=\inf \{\sigma(x, y \circ h) \mid h$ is a sensepreserving homeomorphism of $I$ onto $J\}$. Then $D_{P}$ is a pseudo-metric and is a metric on the space of Fréchet equivalence classes: $x F y$ if $D_{F}(x, y)=0$. Such an equivalence class is a Fréchet curve. If $m$ is continuous and monotonically nondecreasing from $I$ onto $J$ then $z F x$ where $z$ is defined by $x=z \circ m$. It is easy to see that $\left|\mu_{n} x-\mu_{n} y\right|$ $\leqq 2 n D_{F}(x, y)$ and $\left|\mu_{x}-\mu_{y}\right| \leqq 4 D_{F}(x, y)$. If $\xi$ and $\eta$ are Fréchet curves with $x \in \xi$ and $y \in \eta$, then let $\mu_{n} \xi=\mu_{n} x, \mu \xi=\mu x$ and $D_{P}(\xi, \eta)=D_{F}(x, y)$.

Lemma 6. If $X, Y \in C_{\mu}$ and $X F Y$, then $X=Y$.

Proof. There exist homeomorphisms $\left\{h_{k}\right\}$ such that $\sigma\left(X, Y \circ h_{k}\right)$ $<k^{-1}$. If $u \in[0,1]$ then $(\mu X)\left|u-h_{k}(u)\right|=\left|\phi_{X}(u)-\phi_{Y}\left(h_{k}(u)\right)\right|<4 k^{-1}$ so that $h_{k}$ converges uniformly to the identity on $[0,1]$.

If $x \in C(I)$, let $X=x \circ \phi x / \mu x$ if $x$ is not constant, and let $X=x(a)$ otherwise. Thus, by some earlier remarks, $X F x$.

Lemma 7. If $x \in C(I)$, then $X \in C_{\mu}$.

Proof. If $u \in[0,1]$, there exists $c \in I$ such that $(\mu x) u=\phi_{x}(c)$. Thus $(X \mid[0, u]) F\left(x \mid I_{c}\right)$ so that $\phi_{x}(u)=\phi_{x}(c)=(\mu x) u=(\mu X) u$.

It follows that each Fréchet curve has a unique representation in $C_{\mu}$.

Let $F$ be the space of Fréchet curves metrized by $D_{F}$. If $x, y \in \xi \in F$ then range $x=$ range $y$. Let $[\xi]$ denote range $x$. 
THEOREM. Let $K$ be a compact subset of $M$ and $B \subset F$. If $[\xi] \subset K$ for all $\xi \in B$ then $B$ is sequentially compact if and only if $\lim _{n \rightarrow \infty} n^{-1} M_{n}=0$ where $M_{n}=\sup \left\{\mu_{n} \xi \mid \xi \in B\right\}$. If $\left[\xi_{n}\right] \subset K$, then $\left\{\xi_{n}\right\}$ contains a convergent subsequence if and only if $\lim _{n \rightarrow \infty} n^{-1}\left(\mu_{n} \xi_{n}\right)=0$.

\section{REFERENCES}

[F] M. Fréchet, Sur quelques points du calcul fonctionnel, Rend. Circ. Mat. Palermo 22 (1906), 1-74.

[M] M. Morse, A special parametrization of curves, Bull. Amer. Math. Soc. 42 (1936), 915-922.

[S] E. Silverman, $A n$ intrinsic property of Lebesgue area, Riv. Mat. Univ. Parma 2 (1951), 195-201.

Purdue University 